\title{
THEORETICAL AND EXPERIMENTAL STUDY OF THE RADIATIVE DECAY PROCESS IN A MODULATION DOPED GaAs/AlGaAs HETEROINTERFACE
}

\author{
T. Lundström, J.P. Bergman, P.O. Holtz, B. Monemar \\ Department of Physics and Measurement Technology, Linköping University \\ 58183 Linköping, Sweden
}

\author{
K. Campman, J.L. Merz and A.C. Gossard
}

QUEST Center, University of California at Santa Barbara

Santa Barbara, CA 93106, USA

\begin{abstract}
We have theoretically and experimentally investigated the radiative recombination process in an $n$-type modulation doped $\mathrm{GaAs} / \mathrm{Al}_{0.35} \mathrm{Ga}_{0.65} \mathrm{As}$ heterostructure. The dynamical reshaping of the potential profile across the heterojunction, and the decay of the spatially indirect radiative recombination between electrons in the two-dimensional electron gas and photo-created holes, have been numerically simulated for various values of the electric field across the heterojunction. Optical matrix elements were deduced from a self-consistent solution of the coupled Schrödinger and Poisson equations at every discrete point of time. The calculated recombination energies and integrated luminescence intensities were compared with experimental data from time-resolved photoluminescence measurements on an $800 \AA$ wide GaAs/ AlGaAs heterostructure.
\end{abstract}

PACS numbers: 78.66.-w, 78.60.-b

\section{Introduction}

By the technique of modulation doping in heterostructures, it is possible to spatially separate the charge carriers from the dopant atoms. The separation will drastically reduce the impurity related scattering of the carriers, and thus result in a very high carrier mobility, e.g. as utilised in the high electron mobility transistor (HEMT). In the case of an $n$-type modulation-doped GaAs/AlGaAs single heterojunction, the donated electrons from the doping in the $\mathrm{AlGaAs}$ barrier are transferred to the GaAs well lateral until thermal equilibrium is reached. The electrons accumulated in the active GaAs region are confined in a narrow notch 
potential close to the heterointerface. The radiative recombination involving the electrons in this quasi two-dimensional electron gas (2DEG) has been extensively studied [1-7].

The recombination involving these electrons (hereafter denoted HB) is characterized by a strong spectral dependence on the experimental conditions, such as the laser excitation power and an externally applied electrical field (gate voltage).

The HB emission is usually [1-7] described as the spatially indirect recombination between an electron from the 2DEG in the notch potential and a photo-created hole at some distance from the 2DEG. In the PL spectra it is observed as an emission band at an energy position between the GaAs bulk excitons and the donor acceptor pair (DAP) recombination, i.e. between $1.51 \mathrm{eV}$ and $1.49 \mathrm{eV}$. In this model, the $\mathrm{HB}$ characteristics, such as peak position and temporal behaviour, strongly depend on the band bending and time evolution of the band bending across the heterojunction. In other words, one important issue in understanding the $\mathrm{HB}$ emission is to have a realistic description of the band bending in the structure. So far, no detailed investigation of the transient behaviour of the $\mathrm{HB}$ emission in a modulation doped GaAs/AlGaAs heterojunction, including a realistic description of the temporal evolution of the band bending, has been attempted to the best of our knowledge.

In this work we theoretically model the time evolution of the HB emission and the band bending across the heterostructure, in an $800 \AA$ wide modulation doped GaAs/AlGaAs heterojunction. The theoretical results are compared with time-resolved photoluminescence (PL) measurements on a heterostructure with the same characteristics.

\section{Experimental details}

The sample used in this study was grown by molecular beam epitaxy (MBE) on semi-insulating GaAs substrates. A GaAs buffer layer and a GaAsAlAs superlattice was grown on the substrate to provide a smooth surface for the active layers. These consisted of a $800 \AA$ wide GaAs layer, a $200 \AA$ wide $\mathrm{Al}_{0.35} \mathrm{Ga}_{0.65}$ As spacer and a $800 \AA$ wide donor doped ( $\left.\mathrm{Si} 10^{18} \mathrm{~cm}^{-3}\right) \mathrm{Al}_{0.35} \mathrm{Ga}_{0.65} \mathrm{As}$ barrier. Finally a $50 \AA$ thick GaAs cap layer was grown on the $\mathrm{Al}_{0.35} \mathrm{Ga}_{0.65} \mathrm{As}$ layer, in order to protect the $\mathrm{Al}_{0.35} \mathrm{Ga}_{0.65}$ As from oxidation. On top, a semitransparent Schottky-gate was evaporated and thin electrical wires were connected.

The luminescence measurements were performed at $2 \mathrm{~K}$ using picosecond pulses from a dye-laser synchronously pumped by a mode locked Ar laser. The laser wavelength was below the band gap of the AlGaAs barrier, and the samples were excited with an unfocused laser beam to obtain an homogeneous excitation intensity. The average laser power density was approximately $0.13 \mathrm{~W} / \mathrm{cm}^{2}$. The luminescence was detected using time-correlated photon counting with a time resolution of $200 \mathrm{ps}$. Time-delayed spectra were obtained by measuring the luminescence decay at various fixed detection wavelengths.

\section{Experimental results}

In Fig. 1a we show typical results from time-resolved PL measurements on the $800 \AA$ wide GaAs/AlGaAs heterostructure. The time delay between the 20 first 

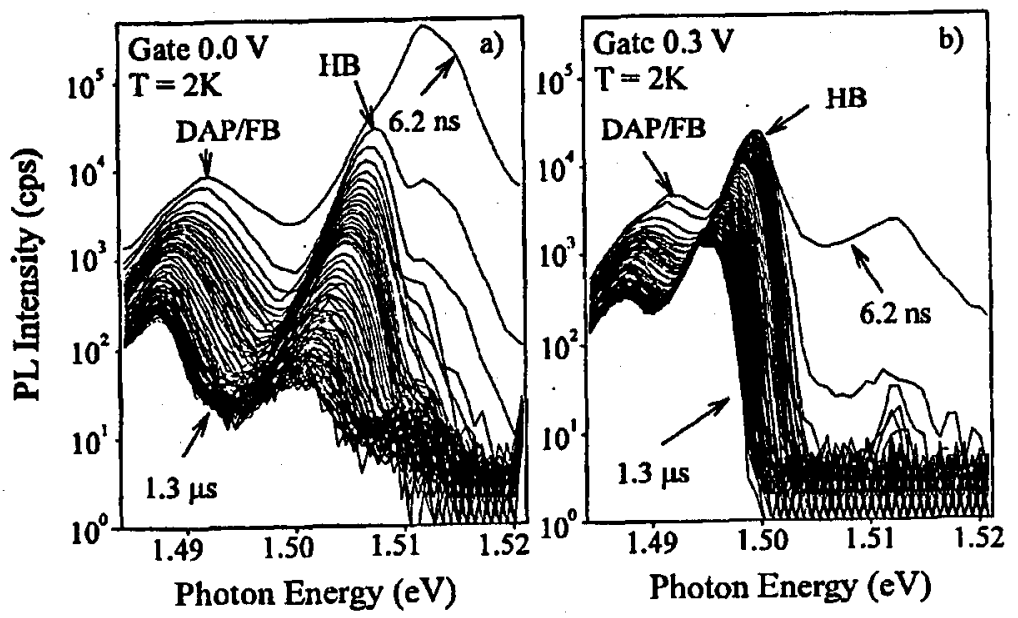

Fig. 1. Time delayed PL spectra at $2 \mathrm{~K}$ for the $800 \AA$ wide GaAs/AlGaAs heterostructure in the time range from $6.2 \mathrm{~ns}$ to $1.3 \mu \mathrm{s}$. The applied gate voltage is (a) $0 \mathrm{~V}$ and (b) $0.3 \mathrm{~V}$.

spectral curves is $6.2 \mathrm{~ns}$, followed by every fourth recorded spectrum. The peak at $1.512 \mathrm{eV}$ exhibits a rapid decay and disappears within the first $30 \mathrm{~ns}$ after the laser pulse excitation. This peak probably involves contributions from several different recombination processes, such as free and bound exciton recombination in the GaAs buffer layer and recombination related to higher electron states in the 2DEG. The broad emission band centred around $1.492 \mathrm{eV}$ is the free-to-bound (FB) and donor acceptor pair (DAP) recombination of bulk GaAs.

The emission band, peaking at $1.507 \mathrm{eV}$ shortly after the laser pulse, we identify as the HB emission. This peak exhibits a dynamical spectral behaviour and shifts slowly towards $1.500 \mathrm{eV}$ with increasing delay time after the laser pulse. With a positive voltage of $0.3 \mathrm{~V}$ (Fig. $1 \mathrm{~b}$ ) applied over the top semi-transparent gate and the substrate (keeping the top gate at a positive potential relative to the substrate), the HB emission is shifted towards lower recombination energies.

From the time resolved PL measurements we have extracted the peak position and integrated intensity of the HB emission by fitting an asymmetric peak shape to the HB emission and a Gaussian peak shape to the DAP and FB emission band (hereafter denoted DAP/FB).

\section{Theoretical calculations}

The subband energy levels and envelope-wave functions are calculated self-consistently by solving the one-dimensional Schrödinger and Poisson equations

$$
\begin{aligned}
& \left\{-\frac{\hbar^{2}}{2} \frac{\mathrm{d}}{\mathrm{d} z} \frac{1}{m^{\mathrm{e}, \mathrm{h}}(z)} \frac{\mathrm{d}}{\mathrm{d} z}+V^{\mathrm{e}, \mathrm{h}}(z)\right\} \Psi_{i}^{\mathrm{e}, \mathrm{h}}(z)=E_{i}^{\mathrm{e}, \mathrm{h}} \Psi_{i}^{\mathrm{e}, \mathrm{h}}(z), \\
& \nabla \varepsilon_{0} \varepsilon_{r}(z) \nabla \Phi_{\mathrm{sc}}(z)=e\left(\sum_{\text {occ }} n_{i}\left|\Psi_{i}^{\mathrm{e}}(z)\right|^{2}-\sum_{\text {occ }} p_{i}\left|\Psi_{i}^{\mathrm{h}}(z)\right|^{2}\right) .
\end{aligned}
$$


Here the occupancy $n_{i}\left(p_{i}\right)$ of electron (hole) subband $i$ at the temperature $T$ is given by the Fermi-Dirac distribution. The electron (hole) quasi-Fermi level $\mu_{\mathrm{e}}\left(\mu_{\mathrm{h}}\right)$ is adjusted to preserve the total electron (hole) density $n_{\mathrm{tot}}\left(p_{\mathrm{tot}}\right) . V_{\mathrm{e}}(z)$ and $V_{\mathrm{h}}(z)$ are the effective electron and hole potential. $\Phi_{\mathrm{sc}}(z)$ is the self-consistent Hartree mean Coulomb field. Many particle corrections to the 2DEG single particles states are taken into account by including a local exchange-correlation potential.

The time evolution of the carrier recombination process after excitation with a short laser pulse are calculated numerically by solving rate equation for the decrease in both the photo-created non-equilibrium electron and hole densities $n_{\text {noneq }}, p_{\text {noneq }}$ :

$$
\frac{\mathrm{d} n_{\text {noneq }}}{\mathrm{d} t}=\frac{\mathrm{d} p_{\text {noneq }}}{\mathrm{d} t}=-\int_{0}^{\infty} R_{\mathrm{sp}}^{i, j}(E) \mathrm{d} E-\frac{1}{\tau_{\mathrm{NR}}} p_{\text {noneq }},
$$

where the sum runs over all electron $i$ and hole $j$ subbands. Note that index $j$ runs over both the heavy and light hole subbands. $R_{\mathrm{sp}}^{i, j}$ is the spontaneous radiative recombination rate with strict $k$-conservation [8]. Nonradiative recombination is introduced as a reduction of the non-equilibrium hole concentration.

\section{Results}

In Fig. 2 we show a comparison between the time evolution of the measured IIB peak position and the calculated E2-IIIH1 recombination energies for different gate voltages. The calculated recombination energies are slightly too high and have been downshifted by $5 \mathrm{meV}$ to ease the comparison. The time evolution of the

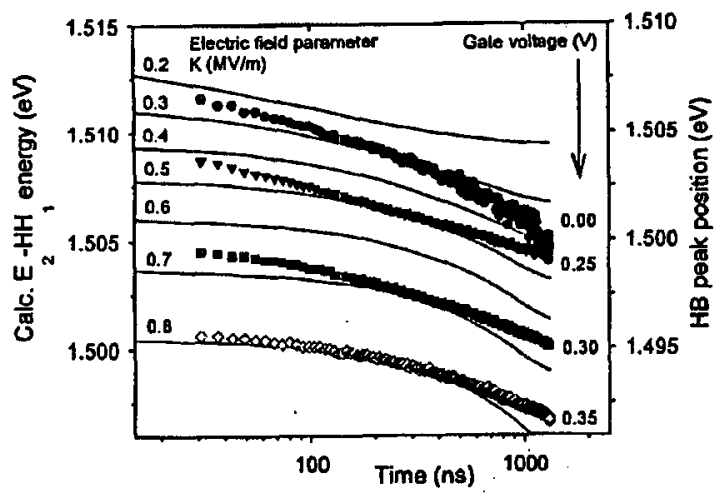

Fig. 2. Calculated recombination energies (lines) for the E2-HH1 transition under various electric fields. Measured HB peak positions are shown with different symbols for positive gate voltage of $0.0,0.25,0.30$, and $0.35 \mathrm{~V}$.

calculated recombination energies shows the same general trend as the measured HB energies, with a relatively larger and faster red shift for small electric field values, than for larger values. The close correspondence between the relative energy shifts of the calculated and measured recombination energies is encouraging. 


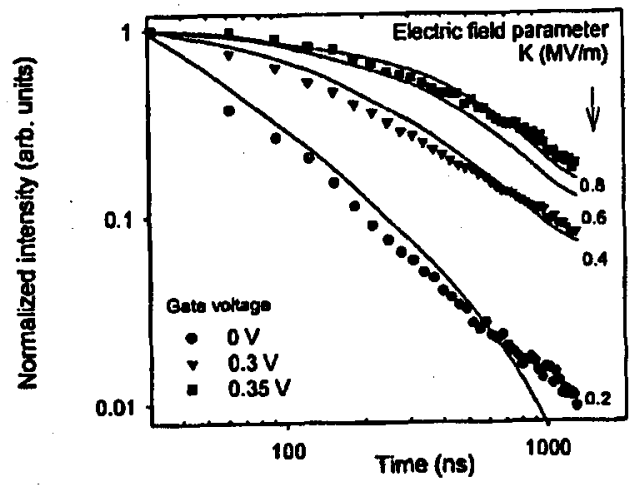

Fig. 3. Normalized calculated PL intensity for various electric field values and normalized measured integrated intensity for the IIB emissions at various applied positive gate voltages.

A similar comparison of the integrated IIB intensity versus time is seen in Fig. 3. In this case we have normalized the intensity to unity at short times after the excitation pulse. As can be seen the calculated intensity exhibits the same general behaviour as the measured IIB intensity.

\section{References}

[1] Y.R. Yuan, K. Mohammad, M.A.A. Pudensi, J.L. Merz, Appl. Phys. Lett. 45, 739 (1984).

[2] Q.X. Zhao, J.P. Bergman, P.O. Holtz, B. Monemar, C. Hallin, M. Sundaram, J.L. Merz, A.C. Gossard, Semicond. Sci. Technol. 5, 884 (1990).

[3] Q.X. Zhao, Y. Fu, P.O. Holtz, B. Monemar, J.P. Bergman, K.A. Chao, M. Sundaram, J.L. Merz, A.C. Gossard, Phys. Rev.B 43, 5035 (1991).

[4] L.M. Weegels, J.E.M. Haverkort, M.R. Leys, J.H. Wollter, Phys. Rev. B 46, 3886 (1992).

[5] J.P. Bergman, Q.X. Zhao, P.O. Holtz, B. Monmear, M. Sundaram, J.L. Merz, A.C. Gossard, Phys. Rev. B 43, 4771 (1991).

[6] I.V. Kukushkin, R.J. Haug, K. von Klitzing, K. Eberl, K. Tötemeyer, Phys. Rev. $B$ 50, 11259 (1994).

[7] T. Lundström, Ph.D. Thesis, Linköping University, Linköping 1997.

[8] R.H. Yan, S.W. Corzine, L.A. Coldren, I. Suemune, IEEE J. Quantum Electron. 26, 213 (1990). 